\title{
Path Integral Monte Carlo Methods: Static- and Time-Correlation Functions
}

\author{
Bruce J. Berne ${ }^{1}$
}

\begin{abstract}
Path integral methods for simulating the structure, thermodynamic properties, and time-dependent response of simple quantum systems are reviewed. These methods are used to simulate the structure of an excess electron in a helium bath.
\end{abstract}

\section{INTRODUCTION}

Feynman's path integral formulation of quantum statistical mechanics ${ }^{(1)}$ makes possible the computer simulation of quantum many-body systems of chemical and physical interest. ${ }^{(2)}$ According to this formulation ${ }^{2}$ the canonical partition function is

$$
Q(\beta, V)=\int d x_{1} \int_{x_{1}}^{x_{1}} D x(\tau) e^{-S[x(\tau)]}
$$

where

$$
S[x(\tau)]=\int_{0}^{\beta \hbar} d \tau H[x(\tau)]
$$

is the Euclidian action corresponding to the path $x(\tau)$ in Euclidean (or imaginary) time $\tau ; H[x(\tau)]$ is the path dependence of the Hamiltonian;

Supported by grants from NSF and NIH. The work reported here was partially supported by a grant of supercomputer time on the University of Minnesota Cray 1 by the OASC of NSF.

${ }^{1}$ Department of Chemistry, Columbia University, New York, N.Y. 10027.

${ }^{2}$ For simplicity we discuss particle motion in one dimension. These expressions are easily generalized. Because many questions still remain about the finite temperature simulation of fermions, we omit this from this paper. 
$\int_{x_{1}}^{x_{1}} D x(\tau)[\cdots]$ represents an integration over all paths starting at $x(0)=x_{1}$ and ending at $x(\beta \hbar)=x_{1}$. In the discrete path representation the Euclidean time $\tau$ is discretized in units $\varepsilon=\beta \hbar / P$ where $P$ is an integer and the continuous path $x(\tau)$ is approximated by straight-line paths between neighboring Euclidean times. This allows (1.1) to be expressed as

$$
Q_{p}=\left(\frac{m P}{2 \pi \beta \hbar}\right)^{P / 2} \int \cdots \int d x_{1}, \ldots, d x_{P} e^{-\beta \Phi} P\left(x_{1}, \ldots, x_{P} ; \beta\right)
$$

where $x_{t}=x(t \beta \hbar / P), x_{t+P}=x_{t}$, and

$$
\Phi_{p}\left(x_{1}, \ldots, x_{P} ; \beta\right)=\frac{m P}{2 \beta^{2} h^{2}} \sum_{t=1}^{P}\left(x_{t}-x_{t+1}\right)^{2}+\frac{1}{P} \sum_{t=1}^{P} V\left(x_{t}\right)
$$

Since (1.3) is equivalent to the classical configurational partition function of $P$ classical particles with potential $\Phi_{P}$, the quantum system is said to be isomorphic to a classical $P$ particle cyclic chain polymer in which each particle $t$ interacts with its neighbors $t-1$ and $t+1$ through a harmonic potential with force constant $m P / \hbar^{2} \beta^{2}$, and each particle $t$ experiences the potential $V\left(x_{t}\right) / P$. Clearly $Q_{p}$ is an approximation to the true $Q$. It is easy to prove that $Q_{p}>Q$ and $Q=\lim _{P \rightarrow \infty} Q_{p}$. In path integral simulations one empirically determines and uses that $P$ beyond which the thermodynamic properties do not effectively change. Clearly the lower the temperature the larger $P$ must be.

The classical isomorphism can be simulated by Monte Carlo techniques. ${ }^{(3)}$ This method has already been applied to the study of quantum effects on liquid structure. For example, Thirumalai et al. used pathintegral Monte Carlo (PIMC) to simulate liquid neon; ${ }^{(4)}$ later Wallqvist and Berne used PIMC to simulate quantum effects on the structure of oligomers of water and on liquid water ${ }^{(5)}$. Bartholomew et al. ${ }^{(6)}$ have studied an excess electron in a fluid of $\mathrm{He}$ atoms; Wallqvist et al. have studied an excess electron in water clusters ${ }^{(7)}$ (also see the poster presented by $\mathrm{D}$. Thirumalai in this conference) and in liquid water ${ }^{(7)}$; Rossky ${ }^{(8)}$ has studied liquid water; and Sprik et al. ${ }^{(9)}$ have studied an excess electron in a hard sphere fluid. An alternative approach is to use molecular dynamics to evaluate path integrals (see below) as was done by Parinello and Rahman $^{(10)}$ to simulate an excess electron in a fused salt and recently by Rossky et al. ${ }^{(11)}$ to simulate an excess electron in liquid water. For a comprehensive review see the paper by Berne and Thirumalai cited in Ref. 2.

To use the classical isomorphism, configurations can be sampled from $\exp -\beta \Phi_{p}\left(x_{1}, \ldots, x_{p} ; \beta\right)$ thus generating a trajectory in configuration space. Estimators for various quantum observables can then be averaged over this 
trajectory. Although this appears to be straightforward, ${ }^{(3)}$ complications can arise. For example, if one evaluates the internal energy from

$$
\langle E\rangle_{P}=-\frac{\partial \ln Q_{P}}{\partial \beta}
$$

one finds that this can be expressed as

$$
\langle E\rangle_{P}=\int d x_{1}, \ldots, d x_{P} P\left(x_{1}, \ldots, x_{P} ; \beta\right) \varepsilon_{P}\left(x_{1}, \ldots, x_{P}, \beta\right)
$$

where

$$
P\left(x_{1}, \ldots, x_{p} ; \beta\right)=\frac{1}{Q_{P}}\left(\frac{m P}{2 \beta \hbar^{2}}\right)^{P / 2} e^{-\beta \Phi_{P}\left(x_{1}, \ldots, x_{p} ; \beta\right)}
$$

is the classical isomorphic configurational distribution function and where

$$
\varepsilon_{p}\left(x_{1}, \ldots, x_{p} ; \beta\right)=\frac{P}{2 \beta}-\frac{m P}{2 \beta^{2} \hbar^{2}} \sum_{t=1}^{P}\left(x_{t}-x_{t+1}\right)^{2}+\frac{1}{P} \sum_{t=1}^{P} V\left(x_{t}\right)
$$

is "an" energy estimator. ${ }^{(2)}$ This consists of a part due to the kinetic energy and a part due to the potential energy. It is easy to show that the variance $^{(12)}$

$$
\sigma_{\varepsilon_{P}}^{2}=\left\langle\left(\varepsilon_{P}-\bar{\varepsilon}_{P}\right)^{2}\right\rangle
$$

diverges as $P \rightarrow \infty$. Herman et al. have shown that a Virial theorem applies and that the energy estimator ${ }^{(12)}$

$$
\varepsilon_{P}^{\mathrm{VIR}}=\frac{1}{P} \sum_{t=1}^{P}\left[V\left(x_{t}\right)+\frac{1}{2} x_{t} \frac{\partial V\left(x_{t}\right)}{\partial x_{t}}\right]
$$

does not suffer from these problems. Bartholomew and Berne ${ }^{(13)}$ have shown that similar problems arise in the simulation of a quantum mechanical lattice theory at fixed volume and temperature.

The question of convergence must be addressed. It is important to realize that different properties converge differently as $P$ is varied. For example, for a harmonic oscillator the Helmholtz free energy converges much more rapidly with $P$ than does the internal energy.

Equation (1) can also be cast in the form

$$
Q_{p}=\int d p_{1}, \ldots, d p_{p} \int d x_{1}, \ldots, d x_{p} \exp \left[-\beta H_{\mathrm{eff}}\right]
$$


where

$$
H_{\mathrm{eff}}=\sum_{t=1}^{P} \frac{p_{t}^{2}}{2 m^{\prime}}+\Phi_{p}\left(x_{1}, \ldots, x_{p} ; \beta\right)
$$

Integration over $\left(p_{1}, \ldots, p_{P}\right)$ shows that with the proper choice of $m^{\prime}$ we recover (1.1). In fact $m^{\prime}$ can be adjusted for convenience. This offers a different method for simulating quantum systems. The effective Hamiltonian, $H_{\text {eff }}$, gives rise to classical equations of motion

$$
\begin{aligned}
& \dot{x}_{t}=\partial H_{\mathrm{eff}} / \partial p_{t} \quad t=1, \ldots, P \\
& \dot{p}_{t}=-\partial H_{\mathrm{eff}} / \partial x_{t}
\end{aligned}
$$

This suggests that classical MD methods can be used to simulate the equilibrium properties of quantum systems. ${ }^{(10)}$ Starting with a given state $\left(x_{1}, \ldots, x_{P}, p_{1}, \ldots, p_{P}\right)$ one integrates the equations of motion numerically. If the system is ergodic, averages of estimators over these ergodic trajectories gives corresponding thermodynamic properties. The Hamiltonian flow conserves energy. Thus one ages the system as in classical MD by scaling the velocities until the average kinetic energy is $(p / 2) k T$.

There is one major problem with this approach. Hall and Berne ${ }^{(14)}$ have shown that for large $P$ the Hamiltonian system will be in the KAM regime and the dynamical system will very likely be nonergodic. These authors suggest the following method for circumventing this problem. A classical trajectory is generated for a number of classical time steps after which new momenta are sampled from the Maxwell distribution. This ensures the sampling of all important regions of phase space.

It is important to recognize that the classical trajectories have nothing to do with the real dynamics of a quantum system. The dynamics in this isomorphic system simply provide a simple algorithm for simulating the equilibrium properties of the system. How then can one simulate the real time dynamics of a quantum system? This is a very difficult problem. We have made some progress toward the goal of determining two-point timecorrelation functions using Monte Carlo methods. ${ }^{(15-17)}$ This makes possible the determination of spectral line shapes and transport properties in condensed systems.

In this lecture I will outline two methods for simulating the real time decay of two-point time-correlation functions, omitting details. The interested reader should consult the references. I will also present one of many equilibrium studies made by my group, namely, that of a single excess electon dissolved in an He fluid of the atoms with which it interacts through a pseudopotential. It is shown that at low fluid densities the elec- 
tron is delocalized whereas at high fluid densities it is localized in a bubblelike state.

\section{TIME-CORRELATION FUNCTIONS IN QUANTUM SYSTEMS}

Time-correlation functions play a central role in the theory of dynamic processes in many-body systems. ${ }^{(18,19)}$ Transport properties, chemical reaction rate constants, spectroscopic line shapes, and neutron and light scattering spectra can all be related to well-defined time-correlation functions. In classical many-body systems, Hamiltonian dynamics or stochastic dynamics provide a basis for simulating time-correlation functions. Unfortunately, the calculation of these functions in quantum systems is a formidable problem. In this paper we present a method for calculating timecorrelation functions in quantum and mixed quantum classical systems based on path integral Monte Carlo techniques.

Equilibrium properties of the quantum system can be obtained by solving the equivalent classical problem using standard numerical techniques (e.g., Monte Carlo and MD) as reviewed above. This formulation of the density matrix, which tacitly assumes that paths that result from exchanging two or more particles can be ignored, has now been successfully applied to the calculation of the equilibrium properties of several systems. Extending this procedure to calculate time-correlation functions necessitates inclusion of paths with weights that are nonpositive in character. This arises because in the expression for time-correlation functions both imaginary (thermal) times and real times are simulataneously present. Direct application of Monte Carlo leads to large fluctuations and thereby to very inaccurate results. We have suggested two different methods for circumventing this problem. ${ }^{(15-17)}$ In one method one defines a symmetrized time correlation that lends itself to direct PIMC or PIMD simulation. ${ }^{(16,17)}$ In the second method we simulate the system using imaginary-time propagators only, and the real-time behavior can then be inferred by analytic continuation. ${ }^{(15)}$ This is very similar to the analytic continuation used to determine properties of real-time Green's functions in $\mathrm{N}$-body quantum systems from the knowledge of the corresponding imaginary-time (finite temperature) Green's functions. ${ }^{(20)}$

It is well-known from linear response theory that the response of a system to a weak external field can be expressed in terms of a timecorrelation function of a dynamical property of the system. ${ }^{(18,19)}$ For example, the infrared absorption spectra is explicable in terms of the dipole-dipole correlation function, and the translational diffusion coefficient is related to the velocity autocorrelation function, etc. In this section we provide a general method to calculate the autocorrelation function 
$\langle A(t) A(0)\rangle$ where $A$ can, in principle, be any quantum mechanical operator representing the $N$-body system. To be specific, let us consider a many-body system consisting of $N$ particles. The Hamiltonian for the system is taken to be

$$
H=\sum_{i=1}^{N} \frac{p_{i}^{2}}{2 m}+U\left(\left\{r_{i}\right\}\right)
$$

where $p_{i}$ is the momentum of the $i$ th particle, $U\left(\left\{r_{i}\right\}\right)$ is the potential of interaction between the $N$ particles, and $\left\{r_{i}\right\}$ denotes the collection of all the coordinates of the many-body system.

Consider the two functions

$$
C_{A B}(t)=Q^{-1} \operatorname{tr}\left[e^{-\beta H} A e^{i H t / \hbar} B e^{-i H t / \hbar}\right]
$$

and

$$
G_{A B}(t)=Q^{-1} \operatorname{tr} A e^{-H \tau} B e^{-H \tau^{*}}
$$

where $t$ is the Minkowski (real) time and $\tau=(\beta / 2-i t / \hbar)$ is a complex time. It is easily shown ${ }^{(16)}$ that $\bar{G}_{A B}(\omega)=\exp (-\beta \hbar \omega / 2) \bar{C}_{A B}(\omega)$ so that $C_{A B}(t)$. can be obtained from $G_{A B}(t)$ by Fourier inversion. If $A$ and $B$ are positiondependent operators, then in the coordinate representation

$$
G_{A B}(t)=Q^{-1} \int d x \int d x^{\prime} A(x) B\left(x^{\prime}\right)\left|\left\langle x\left|e^{-H \tau}\right| x^{\prime}\right\rangle\right|^{2}
$$

so that we need only determine the Green's operator $\left\langle x\left|e^{-H \tau}\right| x^{\prime}\right\rangle$. Only for autocorrelation functions is $G_{A A}(t)$ an even real function of time. In a previous paper ${ }^{(21)}$ we showed how such operators can be evaluated using numerical matrix multiplication together with the short time approximations.

As an example, we calculate the diple-dipole correlation function of a proton moving in a bistable potential (see Ref. 16). It is important to note that we are implicitly treating all bound states. This models a proton tunneling in $\mathrm{H}_{2} \mathrm{O}_{2}$ at a temperature of $10 \mathrm{~K}$. For the dipole operator we take $\mu(q)=\mu_{0} \tan h(\alpha q)$ where $\mu_{0}=1$, and $1 / \alpha=0.1 \mathrm{Au}$ is much smaller than the width of the potential.

If only the two lowest states were to contribute at $10 \mathrm{~K}, G_{\mu \mu}(t)=$ $\left.Q^{-1}\left|\mu_{12}\right|^{2} \exp \left[-\beta\left(E_{0}+E_{1}\right) / 2\right] \cos [\Delta \omega) t\right]$, where $\mu_{12}$ is the transition moment and $\Delta \omega=\left(E_{1}-E_{0}\right) / \hbar$ is the tunnel splitting. In Fig. 1 we compare the simulation using $\mathrm{NMM}^{(21)}$ with $(n=6,9,11)$ with the two-state approximation. This is equivalent to a discretized path integral in the primitive algorithm with $P=64,512$ and 2048 , respectively. We note that a 


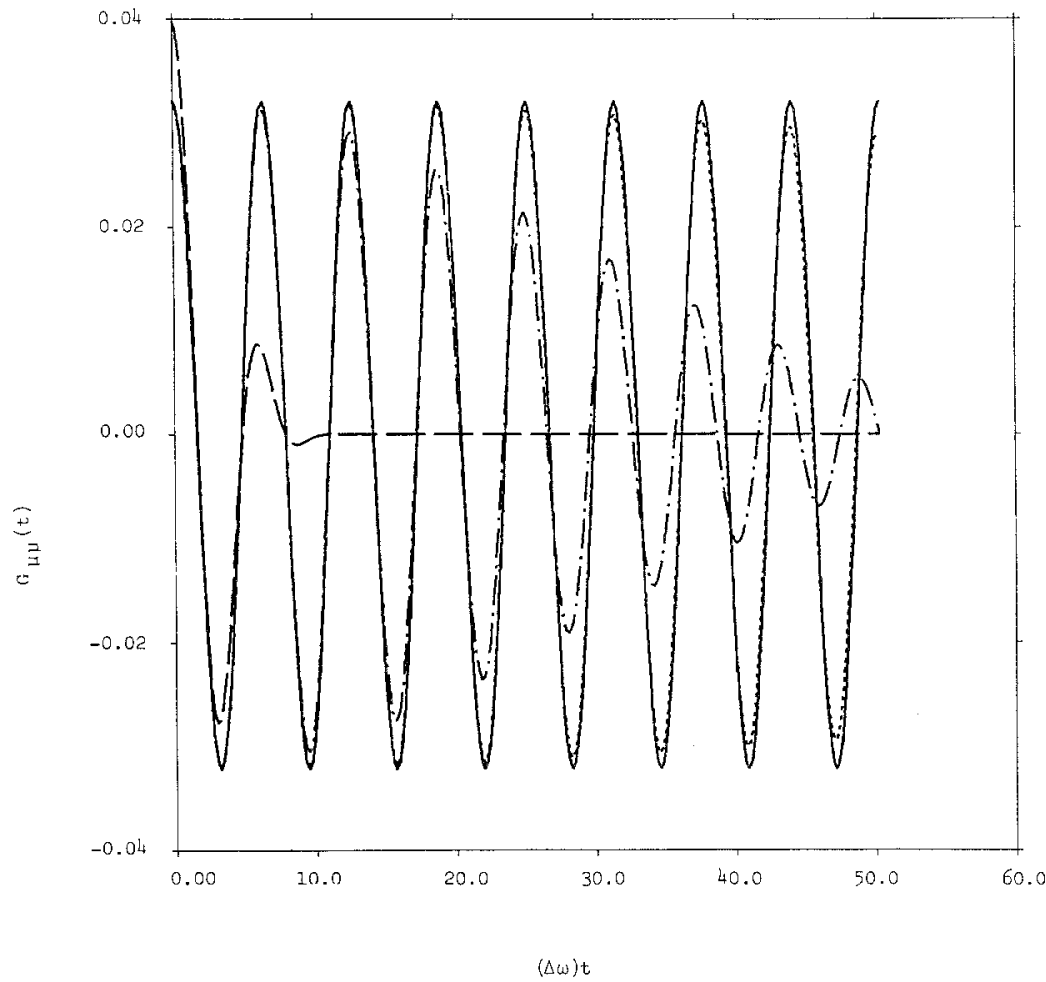

Fig. 1. The correlation function $G_{\mu \mu}(t)$ vs time for a model of protonic tunneling in $\mathrm{H}_{2} \mathrm{O}_{2}$ at $T=10 \mathrm{~K}$. The solid curve represents the analytical time dependence determined using the two lowest states (frequency splitting, $\Delta \omega=4.87 \mathrm{~K}$ ). The curves - - , - . . , , . represent $G_{\mu \mu}(t)$ calculated using discretized path integrals with $P=64,512$, and 2048 , respectively. The time is given in reduced units of $(\Delta \omega) t$, and $G_{\mu \mu}(t)$ is given in atomic units.

large chain is required for good convergence. The time corresponds to eight periods. It is important to note that a similar evaluation of $C_{\mu \mu}(t)$ [cf. (2.2)] leads to numerical instabilities even at short times.

This study shows that it would be inadvisable to use Monte Carlo methods to evaluate $C_{\mu \mu}(t)$. On the other hand, such methods should be stable in the direct evaluation of $G_{\mu \mu}(t)$. This can be appreciated by studying the explicit path integral form of $G_{A B}(t)$

$G_{A B}(t)=\frac{\int d x_{1}, \ldots, d x_{2 P} A\left(x_{1}\right) B\left(x_{P+1}\right) P\left(x_{1}, \ldots, x_{2 P}\right) W\left(x_{1}, \ldots, x_{2 P}\right)}{\int d x_{1}, \ldots, d x_{2 P} P\left(x_{1}, \ldots, x_{2 P}\right) W\left(x_{1}, \ldots, x_{2 P}\right)}$

where $P\left(x_{1}, \ldots, x_{2 P}\right)$ is the Monte Carlo sampling function (and the denominator insures normalization). 


$$
\begin{aligned}
& P\left(x_{1}, \ldots, x_{2 P}\right)=\left(\frac{m^{2} P^{2}}{\pi \hbar^{2}\left(\beta^{2} \hbar^{2}+4 t^{2}\right)}\right)^{P d / 2} \exp \left[-\beta \Phi\left(x_{1}, \ldots, x_{2 P}\right)\right] \\
& \Phi\left(x_{1}, \ldots, x_{2 P}\right)=\sum_{j=1}^{2 P}\left[\frac{m P}{\hbar^{2}\left(\beta^{2} \hbar^{2}+4 t^{2}\right)}\left(x_{j}-x_{j+1}\right)^{2}+\frac{1}{2 P} V\left(x_{j}\right)\right]
\end{aligned}
$$

and where $W$ is

$$
\begin{aligned}
W\left[x_{1}, \ldots, x_{2 P}\right]= & \exp \left[-i t \Omega\left(x_{1}, \ldots, x_{2 P}\right)\right] \\
\Omega= & \sum_{j=1}^{P}\left\{\frac{2 m P}{\left(\hbar^{2} \beta^{2}+4 t^{2}\right)}\left[\left(x_{j}-x_{j+1}\right)^{2}-\left(x_{j+P}-x_{j+P+1}\right)\right]^{2}\right. \\
& \left.-\frac{1}{P}\left[V\left(x_{j}\right)-V\left(x_{j+P}\right)\right]\right\}
\end{aligned}
$$

The basic approach is to sample the configurations $\left(x_{1}, \ldots, x_{2 P}\right)$ from (2.6), evaluate $A\left(x_{1}\right) B\left(x_{P+1}\right)$, and weight its contribution by $W\left(x_{1}, \ldots, x_{2 P}\right)$. Values of $W$ must also be evaluated so that the denominator in (2.5) can be calculated. Because $W$ involves a phase factor that depends on the differences between functions of the sampled configurations, it is expected that cancellation will result and that the average will be much more stable than would be the case for a direct attack on $C_{A B}(t)$. This is borne out in the NMM study given in Fig. 1. Nevertheless, as $t \rightarrow \infty$, the harmonic force constant [cf. (2.6b)] decreases, the polymer becomes delocalized, and the cancellation is less, with comcommitant large phase fluctuations. When there is rapid damping by a bath or only short-time information is required, the phase fluctuations do not cause problems. For nondissipative systems which must be followed for short times, more accurate high temperature approximations for the Greens operator in (2.4) must be employed. Recently we have found that by using a harmonic oscillator reference system with the exact density matrix it will be possible to use this method for nondissipative systems. Recently Behrman and Wolynes has successfully used this method to study real-time correlation functions in a two-level tunneling system coupled to a harmonic bath with an Ohmic density of states. ${ }^{(48)}$

This direct method can also be used to determine electronic absorption and emission spectra at finite temperatures. ${ }^{(17)}$ This offers a simple and more accurate alternative to wavepacket dynamics. ${ }^{(22)}$

In the electric dipole Franck-Condon approximation the cross section corresponding to a transition from electronic state $|i\rangle$ to $|f\rangle$, given an equilibrium thermal distribution of the vibrational states in $|i\rangle$, is

$$
\sigma_{i f}(\omega)=(2 \pi / 3 \hbar c) \omega \bar{C}_{i f}(\omega)
$$


where

$$
\bar{C}_{i f}(\omega)=\frac{1}{2 \pi} \int_{-\infty}^{\infty} d t \exp (i \omega t) C_{i f}(t)
$$

and

$$
C_{i f}(t)=\operatorname{tr} \rho^{(i)} \mu_{i f}(R) \exp \left(i H_{f} t / \hbar\right) \mu_{f i}(R) \exp \left(-i H_{i} t / \hbar\right)
$$

and where the transition dipole moment $\mu_{i f}(R)$ depends on the internal nuclear coordinates $R, H_{i}$, and $H_{f}$ are, respectively, the Hamiltonians of the electronic potential surfaces $|i\rangle$ and $|f\rangle, \quad \rho^{(i)}=\exp \left(-\beta H_{i}\right) /$ $\operatorname{tr} \exp \left(-\beta H_{i}\right)$ is the density operator for the rot $-\mathrm{vib}$ states on the electronic surface $|i\rangle, \beta=(k T)^{-1}$ where $k$ is Boltzmann's constant and $T$ is the temperature, and $\operatorname{tr}(, \ldots$,$) denotes the trace over the rot-vib states.$

Following the approach presented previously we introduce the function

$$
G_{i f}(t)=\operatorname{tr} \mu_{i f}(R) \cdot \exp \left(-H_{f} \tau\right) \mu_{i f}(R) \exp \left(-H_{i} \tau^{*}\right)
$$

where $\tau=\left(\frac{1}{2}\right) \beta-i t / \hbar$ and $\tau^{*}$ is the complex conjugate of $\tau$. It can be shown that $C_{i f}(t)$ and $G_{i f}(t)$ satisfy the relationship

$$
C_{i f}(t)=G_{i f}\left(t-\frac{1}{2} i \beta \hbar\right)
$$

and, consequently, that the Fourier transforms $\bar{C}_{i f}(\omega)$ and $\bar{G}_{i f}(\omega)$ of $C_{i f}(t)$ and $G_{i f}(t)$, respectively, are related by

$$
\bar{C}_{i f}(\omega)=\exp (\beta \hbar \omega / 2) \bar{G}_{i f}(\omega)
$$

As discussed before, $C_{i f}(t)$ is difficult to determine whereas $G_{i f}(t)$ can be determined directly by path-integral techniques. To proceed we express $G_{i f}(t)$ in the position representation

$$
\begin{aligned}
G_{i f}(t)= & \int d R^{\prime} \int d R \mu_{i f}\left(R^{\prime}\right) \cdot \mu_{i f}(R) \\
& \times\left\langle R\left|\exp \left(-H_{f} \tau\right)\right| R^{\prime}\right\rangle\left\langle R^{\prime}\left|\exp \left(-H_{i} \tau^{*}\right)\right| R\right\rangle
\end{aligned}
$$

In previous papers we showed how the Greens operators, $\left\langle R|\exp (-H \tau)| R^{\prime}\right\rangle$, can be determined using an iterative scheme (NMM) starting with the short-time (semiclassical) approximation. ${ }^{(21,17)}$ Once these propagators are determined it is a simple matter to evaluate the spectrum for any function, $\mu_{i f}(R)$, so that the Condon approximation, $\mu_{i f}(R)=\mu_{i f}\left(R_{e}\right)$ need not be made (here $R_{e}$ are the equilibrium positions of the nuclei).

This method has been used to calculate the electronic absorption and emission spectra in a model system with considerable success. (See Ref. 17 
for details.) We believe that it will be particularly useful for the simulation of photochemical reactions.

Another method for calculating quantum mechanical time-correlation functions appears promising. ${ }^{(15)}$ In this method the time-correlation function is calculated by Monte Carlo techniques at several values along the pure imaginary axis of the complex time plane. Using the periodicity of the Euclidean time-correlation function, the real-time behavior is inferred by analytic continuation. This is very similar to the analytic continuation used to determine properties of real-time Greens functions in $N$-body quantum systems from knowledge of the corresponding imaginary-time Greens functions.

The one-sided time correlation functions are defined by

$$
\begin{array}{ll}
F_{>}(t)=\langle A(t) A(0)\rangle=(1 / Q) \operatorname{tr} e^{-\beta H} A(t) A(0) & t>0 \\
F_{<}(t)=\langle A(0) A(t)\rangle=(1 / Q) \operatorname{tr} e^{-\beta H} A(0) A(t) & t<0
\end{array}
$$

where $Q=\operatorname{tr} e^{-\beta H}$. The cyclic invariance of the trace gives rise to

$$
F_{>}(t)=F_{<}(t+i \beta \hbar)
$$

or, equivalently, to detailed balance

$$
f_{>}(\omega)=e^{\beta \hbar \omega} f_{<}(\omega)
$$

where $f_{\gtrless}(\omega)$ are Fourier transforms of $F_{\gtrless}(t)$, respectively.

Let $\lambda$ denote a point on the pure imaginary axis of the complex time such that $0 \leqslant \lambda \leqslant \beta \hbar$. Then for $t=-i \lambda,(2.15)$ can be expressed as

$$
F_{<}(-i \lambda)=(1 / Q) \operatorname{tr} \rho(\beta-\lambda) A \rho(\lambda) A
$$

where $\rho(\gamma)=e^{-\gamma H}$ is the canonical density operator at reciprocal temperature $\gamma^{-1}$. In the position representation this can be expressed as

$$
\begin{aligned}
F_{>}(-i \lambda)= & \frac{1}{Q} \int, \ldots, \int d x_{1} d x_{1}^{\prime} d x_{2} d x_{2}^{\prime} \rho\left(x_{1}, x_{1}^{\prime} ; \beta-\lambda\right) \\
& \times\left\langle x_{1}^{\prime}|A| x_{2}\right\rangle \rho\left(x_{2}, x_{2}^{\prime} ; \lambda\right)\left\langle x_{2}^{\prime}|A| x_{1}\right\rangle
\end{aligned}
$$

The density matrices $\rho\left(x_{1}, x_{1}^{\prime} ; \beta-\lambda\right)$ and $\rho\left(x_{2}, x_{2}^{\prime} ; \lambda\right)$ can now be expressed as path integrals in the usual way

$$
\begin{aligned}
\rho\left(x_{1}, x_{1}^{\prime} ; \beta-\lambda\right) & =\int d x_{2}, \ldots, d x_{N} \prod_{t=1}^{N}\left\langle x_{t}\left|e^{-(\beta-\lambda) H / N}\right| x_{t+1}\right\rangle \\
\rho\left(x_{2}, x_{2}^{\prime} ; \lambda\right) & =\int d x_{N+1}, \ldots, d x_{N+P} \prod_{t=N+2}^{N+P+2}\left\langle x_{t}\left|e^{-\lambda H / P}\right| x_{t+1}\right\rangle
\end{aligned}
$$


where $x_{N+1}=x_{1}^{\prime}, x_{N+2}=x_{2}$, and $x_{N+P+2}=x_{2}^{\prime}$, and where the matrix elements $\left\langle x_{t}\left|e^{-\beta H / P}\right| x_{t+1}\right\rangle$ are replaced by their short-time expansions

$$
\begin{aligned}
\left\langle x_{t}\left|e^{-\beta^{\prime} H / L}\right| x_{t+1}\right\rangle= & {\left[\frac{m L}{2 \pi \hbar^{2} \beta^{\prime}}\right]^{1 / 2} \exp -\left[\frac{m L}{2 \pi \hbar^{2} \beta^{\prime}}\left(x_{t}-x_{t+1}\right)^{2}\right.} \\
& \left.+\frac{\beta^{\prime}}{2 L}\left[V\left(x_{t}\right)+V\left(x_{t+1}\right)\right]\right]
\end{aligned}
$$

Substitution of (2.21) into (2.20a) and (2.20b) gives a path integral expression for $F_{>}(-i \lambda)$, which can be evaluated by direct Monte Carlo simulation for different values of $\lambda$.

To obtain the real-time correlation function $F_{>}(t)$ or, equivalently, its Fourier transform, we note that because $F_{>}(0)=F_{>}(-i \beta \hbar), F_{>}(t)$ can be expanded in a Fourier series

$$
F_{>}(t)=\frac{1}{-i \beta \hbar} \sum_{n} e^{-i Z^{\prime}} n^{\prime} f\left(Z_{n}\right)
$$

where $Z_{n}=2 \pi n /(-i \beta \hbar)$ and $i t=\lambda$. The Fourier coefficients $f\left(Z_{n}\right)$ are

$$
f\left(Z_{n}\right)=\frac{1}{i} \int_{0}^{\beta \hbar} d \lambda \exp \left(Z_{n} \lambda\right) F_{>}(-i \lambda)
$$

where $F_{>}(-i \lambda)$ is determined by PIMC as described above. Now it can be shown that

$$
f(z)=\int_{-\infty}^{+\infty} \frac{d \omega}{2 \pi} \frac{\chi^{\prime \prime}(\omega)}{z-\omega}
$$

is analytic off the real axis and has a branch cut, such that $\chi^{\prime \prime}(\omega)$ is given by the discontinuity across the real axis

$$
\chi^{\prime \prime}(\omega)=\lim _{\varepsilon \rightarrow 0} i[f(\omega+i \varepsilon)-f(\omega-i \varepsilon)]
$$

Here $\chi^{\prime \prime}(\omega)$ is the imaginary part of the susceptibility. Since we can determine $f\left(z=z_{n}\right)$ on the imaginary axis it is possible to analytically continue it into the complex plane. A natural method for performing this analytical continuation is to use the Pade approximant scheme. If the values of $f\left(Z_{n}\right)=f_{n}$ are known for $n=1$ to $n=N+M+1$ values, then $f(z)$ can be represented by the quotient

$$
f(Z)=[N, M]=\sum_{i=0}^{M} b_{i} P_{i}(Z) /\left[q_{0}(Z)+\sum_{i=1}^{N} c_{i} q_{i}(Z)\right]
$$


The coefficients $b_{i}$ and $c_{i}$ can be calculated from the $N+M+1$ values of $\left(Z_{n}\right)$. The functions $P_{i}(Z)$ and $q_{i}(Z)$ are chosen appropriately for the problem being under consideration. This procedure ${ }^{(15)}$ can be summarized:

(1) Evaluate $F_{>}(t)$ along the pure imaginary-time axis

(2) Calculate the Fourier coefficients $f\left(Z_{n}\right)$

(3) Analytically continue $f(Z)$ using Padé approximants

(4) Evaluate $\chi^{\prime \prime}(\omega)$ from $f(Z)$

It should be noted that transport coefficients can be directly related to $f(z=0)$.

So far this method has been applied successfuly to very simple systems. ${ }^{(15)}$ It has yet to be shown that it is useful for complex systems.

\section{BEHAVIOR OF AN ELECTRON IN HELIUM GAS}

The transport properties of an electron in dense gas have been the subject of much experimental and theoretical investigation. In helium, as the gas density increases beyond a certain point, the electron mobility is observed to drop faster than the classical rate. While there is not a pure localization phase transition, a change in the dominant character of the system seems clear.

Feynman, ${ }^{(23)}$ Ferrell, ${ }^{(24)}$ and Kuper ${ }^{(25)}$ have suggested that at high $\mathrm{He}$ density the electron is trapped inside a bubble. Hiroike et al. ${ }^{(26)}$ have calculated the radius and energy of this bubble using a soft electron-He pseudopotential. The radius of the bubble is determined by a compromise between the kinetic energy which delocalizes the electron, the repulsive electron-He potential which localizes the electron, and the free energy required to form the He-bubble interface.

We report the results of a path-integral Monte Carlo simulation of an electron in dense $\mathrm{He}$ gas as a function of $\mathrm{He}$ density using a realistic, "soft" electron-He interaction. ${ }^{(5)}$ Comparison is made with hard-sphere models simulated elsewhere. ${ }^{(27-29)}$ We perform our study at $77.6 \mathrm{~K}$ to make contact with the electric-mobility data of Bartels ${ }^{(30)}$ and Schwartz. ${ }^{(31,32)}$ Our results seem to be consistent with bubble formation.

The Euclidean action for the system is

$$
\begin{aligned}
\Phi \equiv & \sum_{t=1}^{P} \frac{m_{e} P}{2 \hbar^{2} \beta^{2}}\left(\boldsymbol{x}_{t}-\mathbf{x}_{t+1}\right)^{2}+\frac{1}{P} \sum_{j=1}^{N} \sum_{t=1}^{P} V_{e-\mathrm{He}}\left(\left|\mathbf{r}_{j}-\mathbf{x}_{t}\right|\right) \\
& +\sum_{j>i} V_{\mathrm{He}-\mathrm{He}}\left(\left|\mathbf{r}_{j}-\mathbf{r}_{i}\right|\right)
\end{aligned}
$$


where $\mathbf{r}_{1}, \ldots, \mathbf{r}_{N}$ give the position of $N$ HE atoms, $\mathbf{x}_{1}, \ldots, \mathbf{x}_{P}$ give the coordinates of the $P$-point discretized path of the electron, $\beta=\left(k_{B} T\right)^{-1}$, $V_{e-\mathrm{He}}\left(\left|\mathbf{r}_{j}-\mathbf{x}_{t}\right|\right)$ gives the potential between the discrete electron point and the $j$ th $\mathrm{He}$ atom, and $V_{\mathrm{He}-\mathrm{He}}\left(\left|\mathbf{r}_{j}-\mathbf{r}_{k}\right|\right)$ is the interaction potential between the $j$ th and the $k$ th $\mathrm{He}$ atoms. The first two terms in (3.1) represent the potential energy of a classical cyclic chain polymer containing particles of mass $m_{e}$ with nearest-neighbor harmonic bonds. Each bead interacts with each $\mathrm{He}$ atom. In the simulation we take $V_{\mathrm{He}-\mathrm{He}}(r)$ to be a Lennard-Jones (LJ) 12-6 potential with $\sigma=2.576 \AA$ and $\varepsilon=10.22 \mathrm{~K} . V_{e-\mathrm{He}_{\mathrm{e}}}\left(\left|\mathbf{r}_{j}-\mathbf{x}_{t}\right|\right)$ is given by the pseudopotential of Kestner et al. ${ }^{(33)}$ (This potential is valid only at zero momentum, but we do not expect this to be a relativity large source of error.) The potential is maximum ( $0.32 \mathrm{AU})$ at a distance $r=0.61 \AA$ and drops by one-half at $r=1.1 \AA$.

The simulation proceeds as follows. The first term on the right-hand side of (3.1) can be expressed as $\beta \Phi_{e}=\sum_{k} \lambda_{k} Q_{k}{ }^{2} / 2$, where $Q_{k}$ are the normal modes of the chain. We first sample the full set of $(k \neq 0)$ normal modes, the step size of mode $k$ being proportional to $\lambda_{k}{ }^{-1 / 2}$. The Cartesian coordinates are then calculated and the move accepted or rejected using the Monte Carlo algorithm ${ }^{(3)}$ for the full system. The zero-mode translation is made separately. Each He atom is moved separately for the $x, y$, and $z$ coordinates. Spherical bookkeeping is used.

For our study, the polymer chain has $P=100$ beads and the system contains $N=512$ helium atoms. For many runs, an FPS164 Attached Processor (AP) was used; one pass at a helium density of $n=1 \times 10^{22} \mathrm{~cm}^{-3}$ takes $4.8 \mathrm{~s}$. At each density $n$ from 8000 to 15000 passes were made. Additionally, we made three long runs at $n=0.9,1.05$, and $1.25 \times 10^{22} \mathrm{~cm}^{-3}$ of 75000 passes on the Cray 1 ; we obtained a factor 8.7 improvement in time over the AP.

The radial distribution function of $\mathrm{He}$ atoms around the electron barycenter

$$
g_{e-\mathrm{He}}(r) \equiv\left\langle\delta\left(\mathbf{r}-\mathbf{r}_{\mathrm{He}}+\frac{1}{P} \sum_{t=1}^{P} \mathbf{x}_{t}\right)\right\rangle
$$

is determined; this latter quantity is choosen because it can reflect whether or not an electron bubble exists. The nonbarycentric, true distribution function, less suited to signaling bubble formation (where there is no translational invariance) was found not to be as sensitive to changes in density. Also determined are the imaginary-time correlation functions of the form

$$
R_{n}\left(t-t^{\prime}\right) \equiv\left\langle\left|\mathbf{x}_{t}-\mathbf{x}_{t^{\prime}}\right|^{2}\right\rangle^{1 / 2}
$$

at He density $n$. These are the root-mean-square displacements between two polymer points separated by a time $\left(t-t^{\prime}\right) . R_{n}(\beta \hbar / 2)$ represents the 
"diameter" of the polymer chain; this quantity is discussed by Chandler et al. ${ }^{(27,28)}$

In the simulations reported here, the box edge length was usually taken to be at least twice the diameter of the polymer. The action, $\Phi$, was monitored to determine when the system equilibrated. Uncertainties for the quantities measured were determined by blocking the data in successively larger bins until the errors appeared uncorrelated.

Figure 2 shows $R_{n}(t)$ vs $t$ for various He densities $\left[R_{0}(\beta \hbar / 2)=29.2 \AA\right]$. As the density increases the maximum at $t=\beta \hbar / 2$ decreases and the curve levels off faster; the polymer chain goes from a free, extended state to a compact, quasi-localized state showing signs of ground-state dominance. ${ }^{(27,28)}$

Figure 3 shows $R_{n}(\beta \hbar / 2)$ vs density $n$. Initially the decrease is slow. The most rapid decrease is around $n=1.0 \times 10^{22} \mathrm{~cm}^{-3}$ (corresponding to $n \sigma^{3}=0.17$, where $\sigma$ is the LJ parameter); there is a slower decrease at high

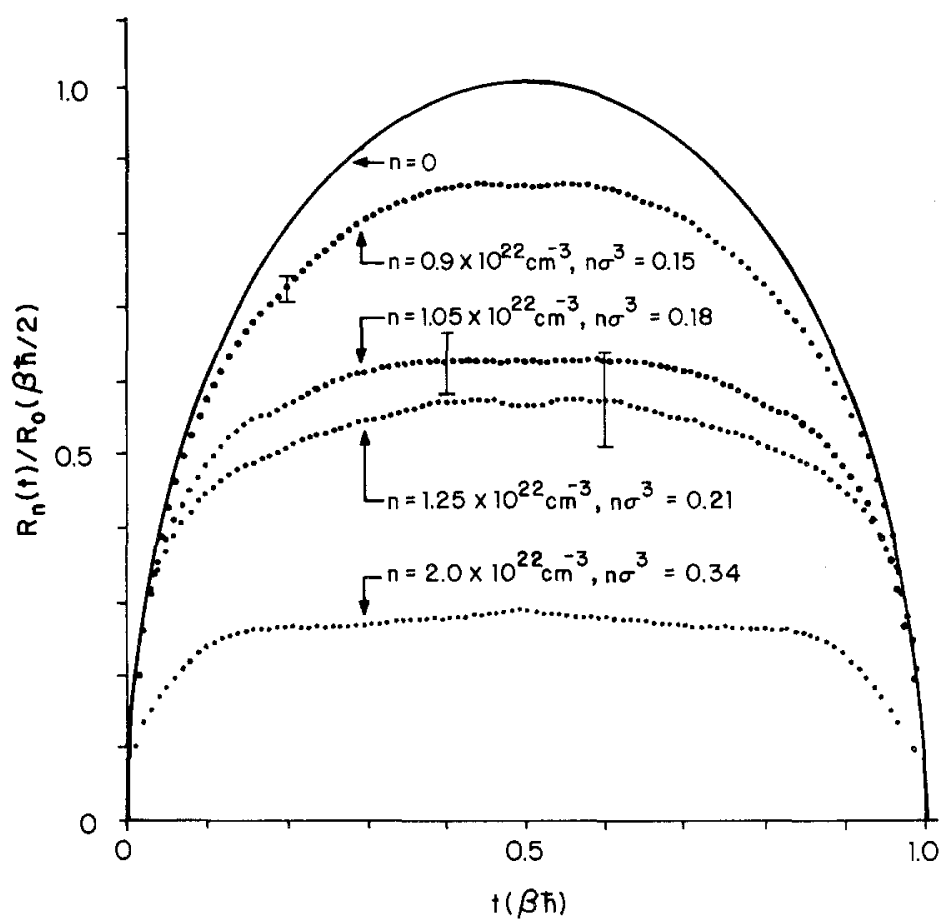

Fig. 2. For the electron-in-helium system at $77.6 \mathrm{~K}$, the correlation function $R_{n}(t)$ vs time $t$ for He densities $n=0,0.9,1.05,1.25$ (all on the Cray), and 2.0 (on the AP) $\times 10^{22} \mathrm{~cm}^{-3}$ scaled to the zero-density value $R_{0}(\beta \hbar / 2) ; \sigma$ is the He-He LJ parameter. The error bars shown are typical (the ones for $n=2.0 \times 10^{22} \mathrm{~cm}^{-3}$ are too small to show). 


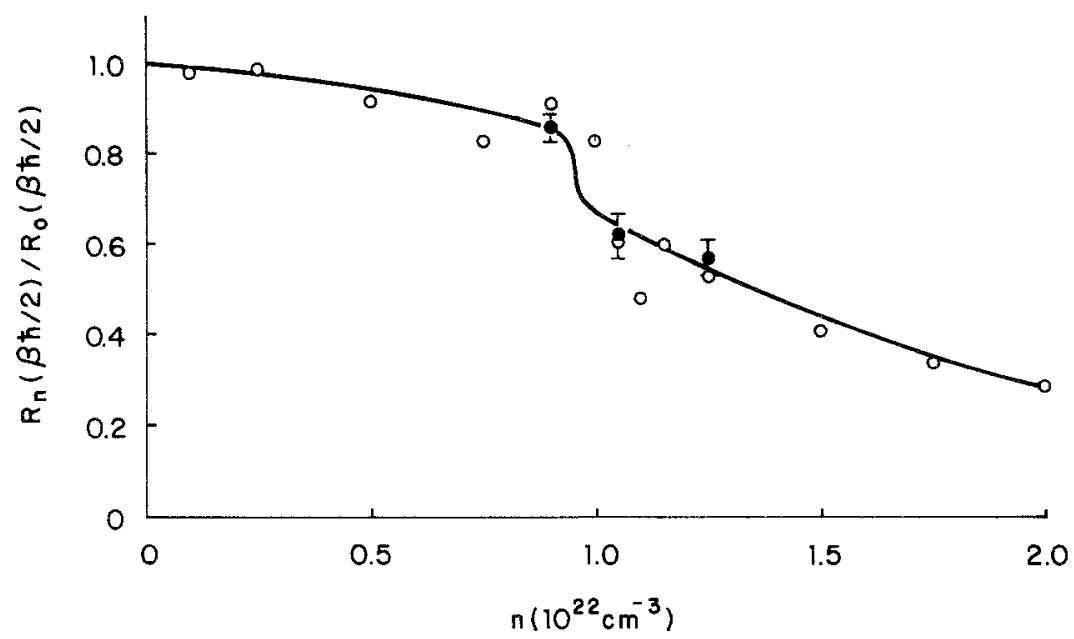

Fig. 3. The electron polymer extent $R_{n}(\beta \hbar / 2)$ scaled to the zero-density value vs density $n$. Cray data are plotted as closed circles, AP data as open circles. The curve is drawn to guide the eye.

densities. For the AP points, error bars cannot be as confidently determined because there are fewer passes. We show these data without error bars, the scatter in the points themselves giving a measure of error. One should not read fine structure into the $R_{n}(\beta \hbar / 2)$ vs $n$ curve; the curve drawn to guide the eye shows the general trend of the data. The Cray runs give high-precision data showing more specifically the shape of the curve and act as a standard for the AP runs.

At $n=1.25 \times 10^{22} \mathrm{~cm}^{-3}$, the polymer behaved as if the electron were localized for $4 \times 10^{4}$ passes with $R_{n}(\beta \hbar / 2) / R_{0}(\beta \hbar / 2)=0.44 \pm 0.02$ and then shifted to a quasi-free state for about $10^{4}$ passes with $R_{n}(\beta \hbar / 2) / R_{0}(\beta \hbar / 2)=1.02 \pm 0.01$. This shows that the electron enters a region where the gas is locally rarefied and remains there metastably.

We may extract a bubble radius, $R=\left(\frac{1}{2}\right) R_{n}(\beta \hbar / 2) \approx(4 \pm 1) \AA$ at high density. This is in excellent agreement with the value $R \approx 4.2 \AA$ given by Jahnke and Silver ${ }^{(34)}$ for He gas at a temperature $T=77.3 \mathrm{~K}$, nearly the temperature used in our simulation. For liquid $\mathrm{He}$ at $T=0 \mathrm{~K}$, Hiroike et al. ${ }^{(28)}$ concluded $R=12.4 \AA$. For the gas at $T \approx 4.2 \mathrm{~K}, R \approx 16 \AA$ by Levine and Sanders, ${ }^{(35)} R \approx 16 \AA$ by Young, ${ }^{(36)} R \approx(6-15) \AA$ by Eggarter and Cohen, ${ }^{(37)} R \approx 14 \AA$ by Eggarter,(38) $R \approx 16 \AA$ by Schwarz. ${ }^{(32)}$ While Jahnke and Silver considered their value low given the liquid He values, it is quite reasonable considering that the bubble radius and other quantummechanical wavelengths will generally scale as $1 / T^{1 / 2}$.

Our decrease in $R_{n}(\beta \hbar / 2)$ with $n$ is slower than that for a rigid, dis- 

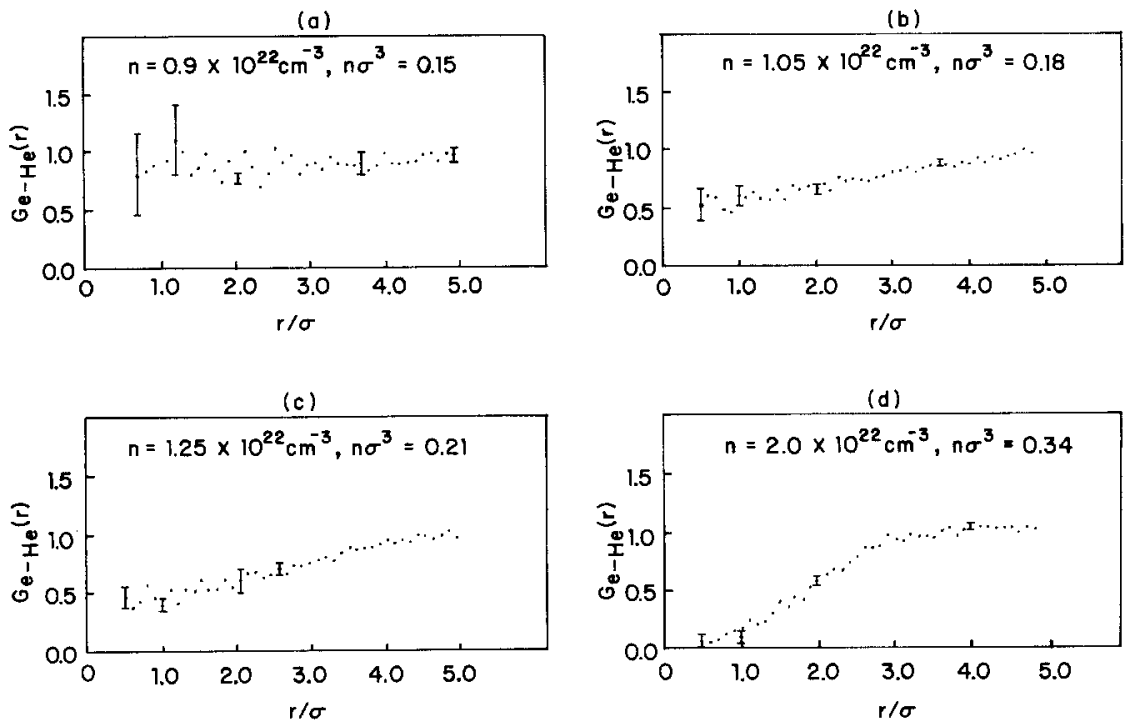

Fig. 4. The electron-helium radial-distribution function $g_{e-\mathrm{He}}(r)$ vs distance $r$ taken with respect to the polymer barycenter at four densities: $n=0.9,1.05,1.25$, and $2.0 \times 10^{22} \mathrm{~cm}^{-3}$. $(\mathrm{a}-\mathrm{c})$ Cray data; (d) AP data. $\sigma$ is the He-He LJ parameter. The error bars shown are typical.

ordered configuration of hard spheres considered by Sprik, Klein, and Chandler. ${ }^{(29)}$ This is very likely a result of their much greater implied temperature which would result in considerable density-scale compression. For a reasonable value of the electron-He distance of closest approach, the implied temperature is several hundred degrees. ${ }^{3}$ Further, the general shapes of the curves are different, there again being the absence of a shoulder.

Figure 4 shows the electron-He radial distribution functions $g_{e-H e}(r)$ taken from the polymer barycenter at four densities. ${ }^{4}$ As the density increases, $\mathrm{He}$ atoms are excluded from the volume occupied by the polymer, resulting in the bubble. At $n=2.0 \times 10^{22} \mathrm{~cm}^{-3}$, we may again read off a bubble radius of $R \approx(5 \pm 1) \AA$ consistent with our earlier determination.

Note that there may be "asynchronization" of static and dynamic properties of the system. The data of Bartels ${ }^{(30)}$ show a much stronger decrease in mobility by $n=0.5 \times 10^{22} \mathrm{~cm}^{-3}$ than any of the static quantities

${ }^{3}$ In Fig. 5 of Ref. 27 , there is a $35 \%$ decrease in $R(\beta \hbar / 2)$ from $n=0$ to $1.05 \times 10^{22} \mathrm{~cm}^{-3}$, taking the electron-He distance of closest approach $\sigma / 2 \approx 1.1 \AA$; the temperature for our problem is $548^{\circ} \mathrm{K}$.

${ }^{4} \mathrm{We}$ do not attempt to smooth these data since only one electron is being considered. 
measured here would indicate; there seems to be a "lag" between static and dynamic measurements. This implication holds also for Ref. 28. As the statics are already difficult, the dynamics will not be easily addressed.

\section{DISCUSSION}

Path integral methods are indeed useful for simulating equilibrium systems. Several problems arise in connection with these methods. In addition to the problems discussed explicitly in this paper, there is the question of convergence. At a given temperature $T, P$ must be taken large enough such that the results do not change significantly for larger $P$. If $P$ is very large the force constants in (1.4) become so large that only very small particle moves are accepted. One is then dealing with a very stiff polymer chain that relaxes very slowly. Several strategies have been adopted to handle this problem.

(1) By transforming the kinetic energy part of the action to normal modes, all modes can be sampled efficiently and, moreover, the zero frequency mode (corresponding to the translation of the whole polymer) can be sampled such that covergence is much more rapid. ${ }^{(5)}$

(2) Fourier path integral techniques can be exploited. ${ }^{(39)}$ If one truncates the number of normal modes, this method is equivalent to the normal mode approach. Recently an algorithm has been discussed that treats the low frequency modes exactly but treats the high frequency modes in a kind of mean field theory. This method looks promising. ${ }^{(40)}$

(3) $P$ can be reduced by using a higher order short-time approximation $^{(41)}$ or by using renormalization group techniques to generate an effective potential at the polymer beads. ${ }^{(44)}$ In some cases $P$ can be reduced by umbrella sampling techniques. ${ }^{(43)}$

(4) Staging algorithms have proven useful. ${ }^{(4)}$ In a staging algorithm one moves a primary polymer chain with relatively small $P$ as though it represents a free quantum particle and accepts or rejects the move by introducing secondary chains between each Euclidean time-adjacent pair of beads.

All of these methods have proven useful in different systems. For example, staging ${ }^{(44)}$ appears to be particularly useful in the study of dilute systems with strong short-range repulsive potentials. Nevertheless, it should not be assumed that because it is particularly effective in treating one kind of system it is naturally superior to other methods when applied to another system. It is clear that much remains to be learned about the simulation of equilibrium systems. 
Time-correlation functions involve even more difficult problems. As pointed out in the text, for large $t$ the force constants get small and the phase fluctuations get large. To avoid this one can use exact local harmonic propagators $^{(45)}$ as a reference system (or other reference systems). This leads to much smaller phase fluctuations. We are currently experimenting with these techniques. ${ }^{(45)}$ The general problem of determining response functions of many-body systems still remains an active field filled with interesting problems.

In this paper I tried to touch on some of the features of simulating quantum systems. This review is not meant to be comprehensive.

\section{REFERENCES}

1. R. P. Feynman and A. R. Hibbs, Quantum Mechanics and Path Integrals (McGraw Hill, New York, 1965).

2. J. A. Barker, J. Chem. Phys. 70:2914 (1979); T. Morita, J. Phys. Soc. Japan 35:98 (1973). For a more comprehensive review of the literature than presented in this paper see B. J. Berne and D. Thirumalai, Ann. Rev. Phys. Chem., 1986 (in press).

3. N. Metropolis, A. W. Rosenbluth, M. N. Rosenbluth, A. H. Teller, and E. Teller, J. Chem. Phys. 21:1087 (1953).

4. D. Thirumalai, R. W. Hall, and B. J. Berne, J. Chem. Phys. 81:2523 (1984).

5. A. Wallquist and B. J. Berne, Chem. Phys. Letts. 117:214 (1985).

6. J. Bartholomew, R. Hall, and B. J. Berne, Phys. Rev. B 32:548 (1985).

7. A. Wallqvist, D. Thirumalai, B. J. Berne, and C. Pangali, (in preparation); A. Wallqvist, D. Thirumalai, B. J. Berne, and C. Pangali, (in preparation).

8. R. A. Kuharski and P. J. Rossky, Chem. Phys. Letts. 103:357 (1985).

9. M. Sprik, M. L. Klein, and D. Chandler, J. Chem. Phys. 83:3042 (1985).

10. M. Parinello and A. Rahman, J. Chem. Phys. 80:860 (1984).

11. P. Rossky, see paper, this issue.

12. M. F. Herman, E. J. Bruskin, and B. J. Berne, J. Chem. Phys. 76:5150 (1982).

13. J. Bartholomew and B. J. Berne, Phys. Rev. D 30:1791 (1984).

14. R. W. Hall and B. J. Berne, J. Chem. Phys. 81:3641 (1984).

15. D. Thirumalai and B. J. Berne, J. Chem. Phys. 79:5029 (1983).

16. D. Thirumalai and B. J. Berne, J. Chem. Phys. 81:2512 (1984).

17. D. Thirumalai and B. J. Berne, Chem. Phys. Letts. 116:471 (1985).

18. See for example B. J. Berne, in Physical Chemistry: An Advanced Treatise, vol. VIII B, H. Eyring, D. Henderson, and W. Jost eds. (Academic, New York, 1971).

19. R. G. Gordon, Adv. Mag. Reson. 3:1 (1968).

20. G. Baym and N. D. Mermin, J. Math. Phys. 2:232 (1961).

21. D. Thirumalai, E. J. Bruskin, and B. J. Berne, J. Chem. Phys. 79:5063 (1983).

22. J. R. Reimers, K. R. Wilson, and E. J. Heller, J. Chem. Phys. 79:4749 (1983).

23. R. P. Feynman, unpublished (reported in C. G. Kuper, Phys. Rev. 122:1007, 1961).

24. R. A. Ferrell, Phys. Rev. 108:167 (1957).

25. C. G. Kuper, Phys. Rev. 122:1007 (1961).

26. K. Hiroike, N. R. Kestner, S. A. Rice, and J. Jortner, J. Chem. Phys. 43:2625 (1965); J. Jortner, N. R. Kestner, S. A. Rice, and M. H. Cohen, J. Chem. Phys. 43:2614 (1965).

27. D. Chandler, Y. Singh, and D. M. Richardson, J. Chem. Phys. 81:1975 (1984). 
28. A. L. Nicholas III, D. Chandler, Y. Singh, and D. M. Richardson, J. Chem. Phys, 81:5109 (1984).

29. M. Sprik, M. L. Klein, and D. Chandler, National Research Council of canada and University of Pennsylvania Reports.

30. A. Bartels, Appl. Phys. 8:59 (1975).

31. K. W. Schwarz, Phys. Rev. Lett. 41:239 (1978).

32. K. W. Schwarz, Phys. Rev. B 21:5125 (1980).

33. N. R. Kestner, J. Jortner, M. H. Cohen, and S. A. Rice, Phys. Rev. A 1:56 (1965).

34. J. A. Jahnke and M. Silver, Chem. Phys. Lett. 19:231 (1973).

35. J. L. Levine and T. M. Sanders, Jr., Phys. Rev. 154:138 (1967).

36. R. A. Young, Phys. Rev. A 2:1983 (1970).

37. T. P. Eggarter and M. H. Cohen, Phys. Rev. Lett. 27:129 (1971).

38. T. P. Eggarter, Phys. Rev. A 5:2496 (1972).

39. J. D. Doll and D. L. Freeman, J. Chem. Phys. 80:2239 (1984); J. Doll, J. Chem. Phys. 81:3536 (1984).

40. R. Coalson and J. Doll, preprint, 1985.

41. H. De Raedt and B. De Raedt, Phys. Rev. A 28:3575 (1983).

42. D. Chandler and R. G. Wolynes, J. Chem. Phys. 74:4078 (1981); K. S. Schweitzer, R. M. Stratt, D. Chandler, and P. G. Wolynes, J. Chem. Phys. 75:1347 (1981).

43. R. A. Friesner and R. H. Levy, J. Chem. Phys. 80:4488 (1984).

44. M. Sprik, M. L. Klein, and D. Chandler, Phys. Rev. B 31:4234 (1985).

45. E. C. Behrman and P. G. Wolynes, J. Chem. Phys. 83:5863 (1985). 